\title{
Application of a Heterogeneous Correlation Integration Method to a Context Cube Network Semantic Model for Railway Passengers
}

\author{
Motoki Yokoyama ${ }^{\mathrm{a}, 1}$, Yasushi KIYOKI ${ }^{\mathrm{b}}$ and Tetsuya MITA ${ }^{\mathrm{a}}$ \\ ${ }^{a}$ Research and Development Center of JR East Group, East Japan Railway Company \\ ${ }^{\mathrm{b}}$ Graduate School of Media and Governance, Keio University, Japan
}

\begin{abstract}
In recent years, with the development of information technology, many cyber-physical systems, in which real space and the information space are linked for data acquisition and analysis, have been constructed. The purpose of constructing a cyber-physical system is to solve and improve social and environmental problems. An important target is the railway space, which aims to provide safe and stable transportation services as part of the social infrastructure. In this paper, we propose a new data model, the "Context Cube Semantic Network", for the railway space and a metric method that employs an integrated scale based on heterogeneous correlations of purpose, sensibility, and distance for the railway space. Furthermore, we constructed a station guidance system that implements the proposed method and evaluates subjects at the station. As a result, we clarified the effectiveness and applicability of the system.
\end{abstract}

Keywords. Context Awareness, Semantic Computing, Data Modeling, Information Retrieval, Cyber-Physical System

\section{Introduction}

In recent years, with the development of information technology, many cyberphysical systems, in which the real space and information space are linked for data acquisition and analysis, have been constructed. The purpose of constructing a cyber physical system is to solve and improve social and environmental problems. An important target is the railway space, which aims to provide safe and stable transportation services as social infrastructure. An information provision system for railway users is important for improving the value of railways.

An important research field and technology for constructing cyber-physical systems is a database system that has functions of retrieving, combining and analyzing information on multimedia in various contexts. The basic function of this system is the application of the Mathematical Model of Meaning (MMM), which is a semantic computing model with a contextual analytical methodology [1]. Kiyoki et al. have proposed a mathematical model of meaning, which is a context-dependent semantic

${ }^{1}$ Motoki Yokoyama, Graduate School of Media and Governance, Keio University, 5322, Endo, Fujisawa, Kanagawa, Japan, 252-0882; E-mail: motokiy@sfc.keio.ac.jp 
associative memory model, and have conducted research on semantic and associative retrieval systems for various data $[2,3,4,5,6]$. Therefore, the application of these models to dynamically correlate various objects in railway space increases the feasibility of creating new services and business.

Today, railway operators have built information-providing systems for railway users to reduce the load of information acquisition [7,8]. Specific examples include a system that displays suspension/delay information on displays installed in stations and trains, a system that displays the positions of trains in real time, and a congestion degree visualization system that uses cameras installed in stations. In these systems, the information acquisition load of users has been improved with the development of cyberphysical systems.

Currently, the diversification of user values in Japan is progressing with the decrease in the birth rate, increase in foreign visitors, and rapid changes in the social environment. An actual problem is the difficulty of providing information to satisfy the user's needs in the conventional information provision for an unspecified majority. To solve this problem, we have proposed a method to dynamically measure the relevance between users and station facilities/stores according to their context. The basis of this approach is the Mathematical Model of Meaning that understands and measures the meaning of data according to the user's context. The features of the proposed method are to construct a spatial, temporal and semantic data model and measure the amount of correlation among the data models and dynamically measure the correlation among the data according to the user's context.

In this paper, we propose a metric method using an integrated scale that is based on heterogeneous correlations of purpose, sensibility, and distance for the railway space. In addition, a station guidance system will be built. The features of the system are described as follows.

(1) Spatial representation using a data model "context cube semantic network" for the railway space.

(2) Measurement and integration of the correlation among context cubes according to time, space, and meaning and the expression of this correlation in a one-dimensional space.

\section{Related Studies}

The information that satisfies the needs of individual passengers must reflect the context that represents the dynamic/static situation and dynamic/static intention of the passenger [9]. Abowd et al. [10] states "We define context as any information that can be used to characterize the situation of an entity, where an entity can be a person, place, or physical or computational object. We define context-awareness or context-aware computing as the use of context to provide task-relevant information and/or services to a user." Many information-providing systems that reflect the user context have been investigated. For example, a study explored the smartphone application search technique by using search keywords that represent the user status.

Yao et al. and Maroulis et al. aim to improve users by building context-aware location recommendation applications. Their method analyzes temporal, locational and social network data that are obtained via location-based social networks that are serviced 
by tensor factorization. This analysis contributes to higher accuracy in the recommendation of locations according to the context of the user. [12,13]

The fundamental difference from the previous research data model is the addition of a new concept that "General" and "Private". As a result, the data model proposed in this study can prevent the disadvantages of data suppliers and eliminate psychological anxiety. The proposed method in this paper is based on the human memory recall model and differs from methods that employ machine learning and neural networks. Studies have shown that humans have two intuitive and logical decision-making systems[14]. The proposed method in this paper is based on a memory recall model that corresponds to these systems.

\section{Approach}

\subsection{Data Model}

Our method can rank the services that satisfied passengers. In this paper, we classify the context of the passenger into the following eight types: Personal Static Situation (PSS), General Static Situation (GSS), Personal Dynamic Situation (PDS), General Dynamic Situation (GDS), Personal Static Intention (PSI), General Static Intention (GSI), Personal Dynamic Intention (PDI) and General Dynamic Intention (GDI). Personal contexts include personal information about passengers and information that is not disclosed by companies. The general contexts are information that is always shared, such as day, time, and weather information. These contexts are utilized to express railway space objects. The structure of each context is shown in Figure 1.

In this paper, we describe objects of railway space, such as passengers, services and facilities, with context cubes (Figure 2.).

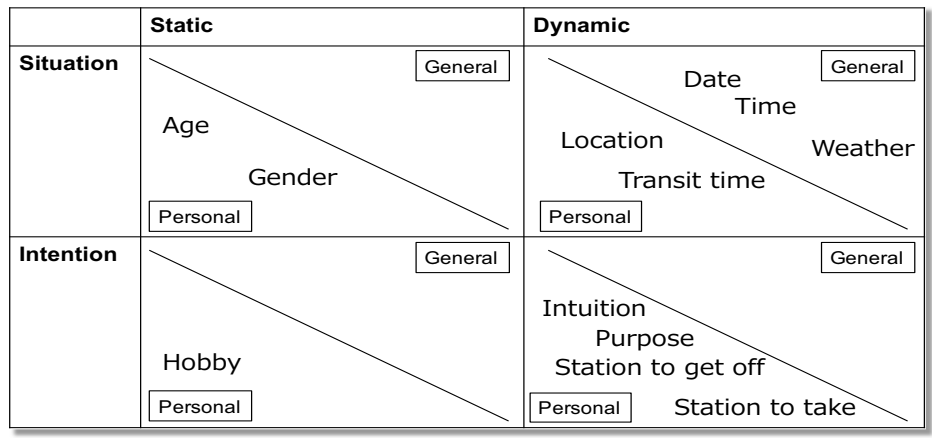

Figure 1. Example of a context 

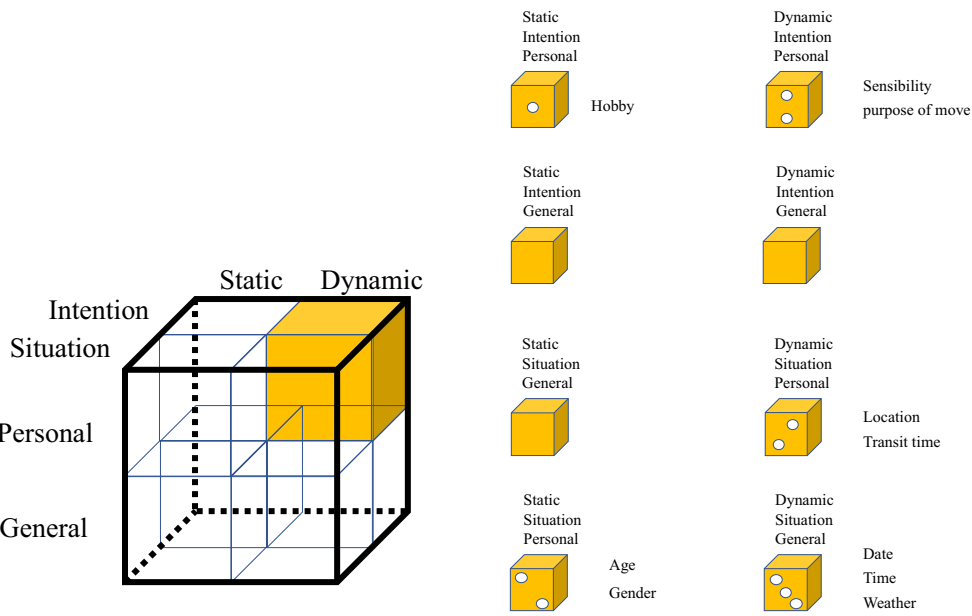

Figure 2. Structure of object context cube

An object can be easily modeled by expressing it in a cube, and various objects can be expressed with the same data model. Both the side that accesses the cube and the cube can be designed to autonomously change its state. The cubes are composed of sub-cubes, which have elements that are contexts, such as passengers and services. The state of a cube changes depending on the state of the railway space and the dynamic interaction among the cubes. Private information can be concealed by target encapsulation.

We dynamically associate entities of the railway space by temporally, spatially and semantically filtering and ordering cubes. Semantic space should be constructed for each object; it will be the subject of weighing relationships, such as those of passengers, facilities and services. For example, in the case of providing information to passengers, a semantic space is constructed from meaningful elements based on passengers, and a cube is mapped to the space. By projecting to the subspace that corresponds to the context, its meaning is determined, and the semantic relation is determined by performing a distance calculation (Figure 3). Furthermore, depending on the temporal and spatial context, whether each cube is included in the ranking can be determined. Specific filtering factors include business hours and ticket gates inside and outside (Figure 4).

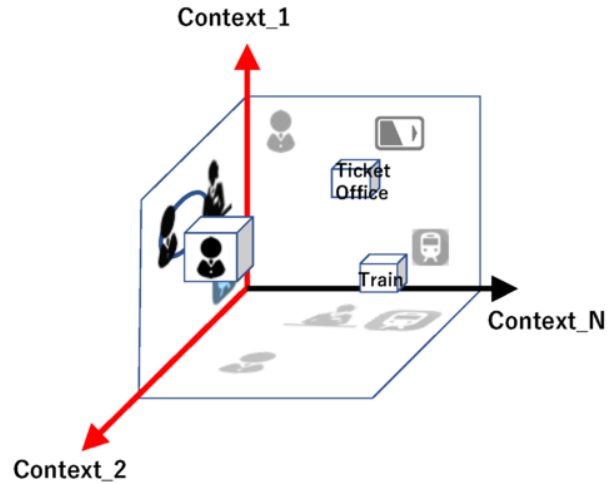

Figure 3.Correlation metric among cubes 


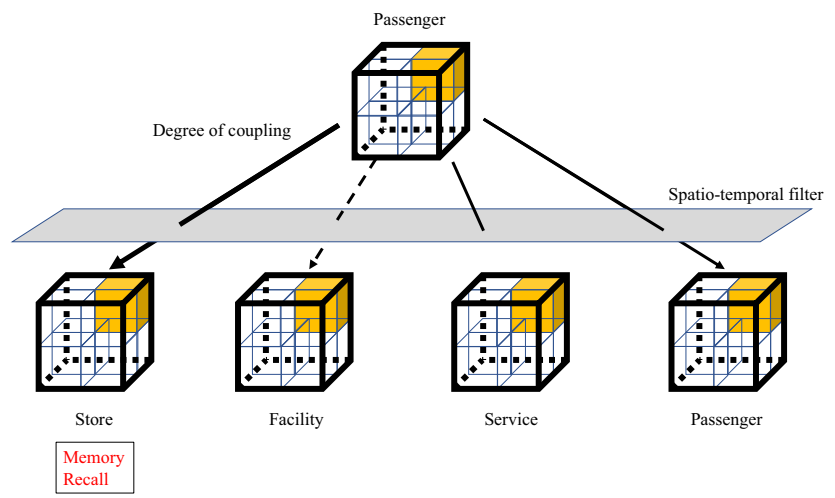

Figure 4. Memory recall by the context cube system

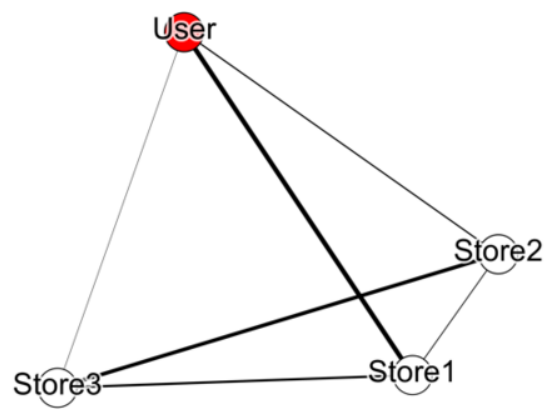

Figure 5. Context Cube Network in Railway Space

In this paper, we propose the Context Cube Semantic Network, which is a semantic network model of the Context Cube, and express the railway space. In this model, a node of a network is a context cube, and a link represents the correlation (weight) between among the context cubes. This model enables context-oriented semantic analysis of the railway space (Figure 5).

\subsection{Correlation Metric Method}

In this paper, we focus on the passenger and establish a correlation metric method among services to enable information provision in anticipation of passenger needs. The preliminary step of passenger needs in this paper is to select a subspace of semantic space based on the intention (purpose) and sense according to the context of passengers, estimate the passenger's context using the correlation measure results for passenger and service. The selection of a space refers to correlation amount weighing, which is an associative model that ignites memory recalls in contexts. The results of the memory recalls that are performed once in a context are different. The procedure of the method is described as follows: 
- Providing Intention Semantic Space $\mathrm{M}_{\mathrm{I}}$ and Sense Semantic Space $\mathrm{M}_{\mathrm{S}}$.

- Providing the Passenger's Context.

- Selecting the Semantic Subspace of $M_{I}$ and $M_{S}$.

- Correlation metric for Passenger's Semantic Subspace $\mathrm{M}_{\mathrm{I}}$ ' and $\mathrm{M}_{\mathrm{S}}$ '.

- Semantic Correlation Integration in 1-dimension.

- Ranking

\subsection{Providing Semantic Space}

The Intention Semantic Space $\boldsymbol{M}_{\boldsymbol{I}}$ set of $\mathrm{m}$ station services (e.g., ticket counter, travel center, etc.) and $\mathrm{n}$ features, in which context elements are utilized in the field of passenger service, is provided in the form of an $\mathrm{m} \times \mathrm{n}$ matrix $\boldsymbol{M}_{\boldsymbol{I}}$ (Figure 6.). Note that the Sense Semantic Space has the same structure as the Intention Semantic Space. In this example, the matrix represents several services that are accessed in the station. These services are characterized using 29 features. $\mathrm{C} 1$ to $\mathrm{C} 15$ in Table 1 are features of the Intention Semantic Space, and the other features pertain to the Sense Semantic Space. If the facility and feature are positively related, the matrix element is 1 ; if they are negatively related, the matrix element is -1 ; otherwise, it is 0 . We define the semantic space $\boldsymbol{M}_{\boldsymbol{I}}$ and $\boldsymbol{M}_{\boldsymbol{S}}$ as the span of the features in Table 1.

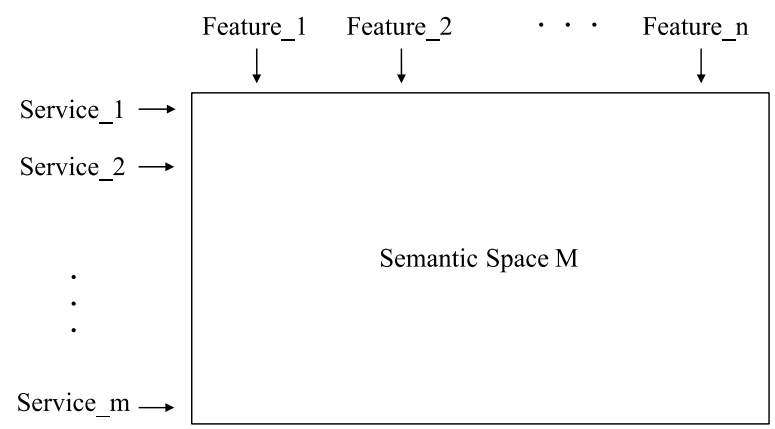

Figure 6. Structure of Semantic Space $\boldsymbol{M}$

Table 1. Abbreviations of context elements

\begin{tabular}{cl}
\hline Abbreviation & \multicolumn{1}{c}{ Feature } \\
\hline $\mathrm{C} 1$ & "Want to buy limited express ticket." \\
\hline $\mathrm{C} 2$ & "Want to buy fare tickets." \\
\hline $\mathrm{C} 3$ & "Want to move smoothly inside the station." \\
\hline $\mathrm{C} 4$ & "Want to take a rest." \\
\hline $\mathrm{C} 5$ & "Want to eat." \\
\hline $\mathrm{C} 6$ & "Want to know various information." \\
\hline $\mathrm{C} 7$ & "Want to use transportation that is not JR." \\
\hline $\mathrm{C} 8$ & "Want to meeting." \\
\hline $\mathrm{C} 9$ & "Want to do shopping." \\
\hline $\mathrm{C} 10$ & "Want to leave a luggage." \\
\hline
\end{tabular}




\begin{tabular}{ll}
\hline C11 & "Want to go to a safe and secure place." \\
\hline C12 & "Want to connect to the Internet." \\
\hline C13 & "Want to pass the time." \\
\hline C14 & "Want to use the Shinkansen." \\
\hline C15 & "Want to prepare a groomed appearance." \\
\hline C16 & "Speedy" \\
\hline C17 & "Feel free" \\
\hline C18 19 & "Fashionable" \\
\hline C20 & "Elegance" \\
\hline C21 & "Recommended" \\
\hline C22 & "Profit" \\
\hline C23 & "Hint" \\
\hline C24 & "Familiar" \\
\hline C25 & "Fun" \\
\hline C26 & "Here, only" \\
\hline C27 & "Enchant" \\
\hline C28 29 & "Lively" \\
\hline "Family"
\end{tabular}

This matrix expresses the space formed by the axes that represent the presence or absence of a feature. Therefore, the services are represented as a vector of the semantic space $\boldsymbol{M}$, as shown in Eq. (1), (Figure 7).

$$
\vec{v}_{\text {service_i }}=\left\{x_{i 1}, \cdots, x_{i j}, \cdots, x_{i n} \mid x_{i j} \in \boldsymbol{M}\right\}
$$

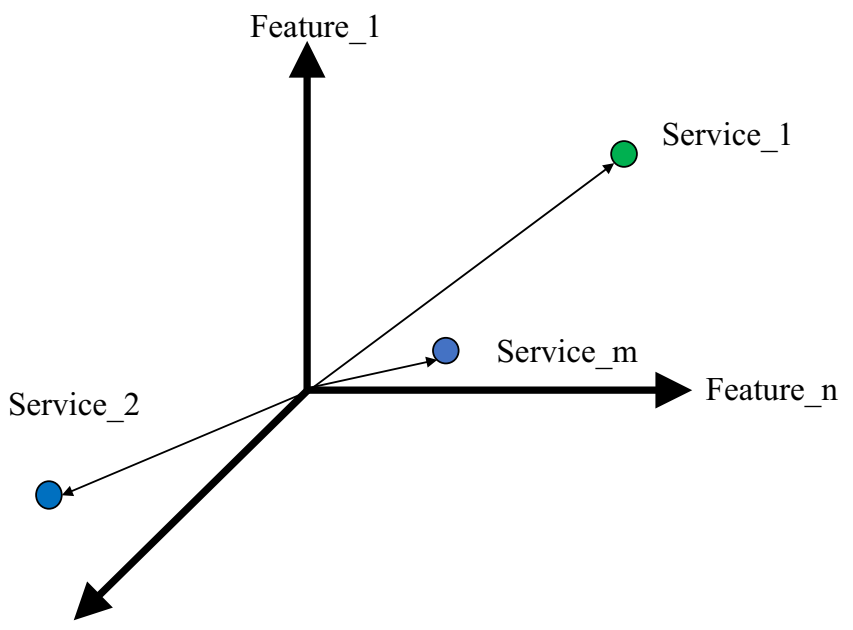

Feature_2

Figure 7. Semantic Space $\boldsymbol{M}$ 


\subsection{Providing the Passenger's Context}

The elements of a passenger's context are provided by smartphones or wearable sensors. In addition, any elements that cannot be acquired from the user are enabled to protect the user's privacy. In this paper, the context is an explicit input from the user interface, such as a button or a list in the smartphone or computer of the user. $\mathbf{U C}$ is the set of user context elements. Context elements include the user vector, user location ul, and time t, etc. The user vector is composed of the intention vector $\vec{v}_{u i}$ and sense vector $\vec{v}_{u s}$. Note that $\vec{v}_{u i}$ and $\vec{v}_{u s}$ have the same structure.

$$
\boldsymbol{U} \boldsymbol{C}=\left\{\vec{v}_{u i}=\left\{y_{1}, \cdots, y .\right\}, \vec{v}_{u s}=\left\{y_{1}, \cdots, y .\right\}, u l, t\right\}
$$

\subsection{Selecting the Semantic Subspace}

The partial space $\boldsymbol{M}^{\prime}$ (semantic subspace) is selected by using features $\mathrm{q}_{i}$, which are beyond the passenger's context, element $y_{i}$, and threshold $\left|\varepsilon_{1}\right|$, in the context (Figure 8).

$$
\boldsymbol{M}^{\prime}:=\operatorname{span}\left(q_{1}, q_{2}, \cdots q_{i}, \cdots, q_{o}\right) \text { where } \mathrm{q}_{i} \in \text { Feature, } y_{i} \geq \text { threshold }\left|\varepsilon_{1}\right| \text { (3) }
$$

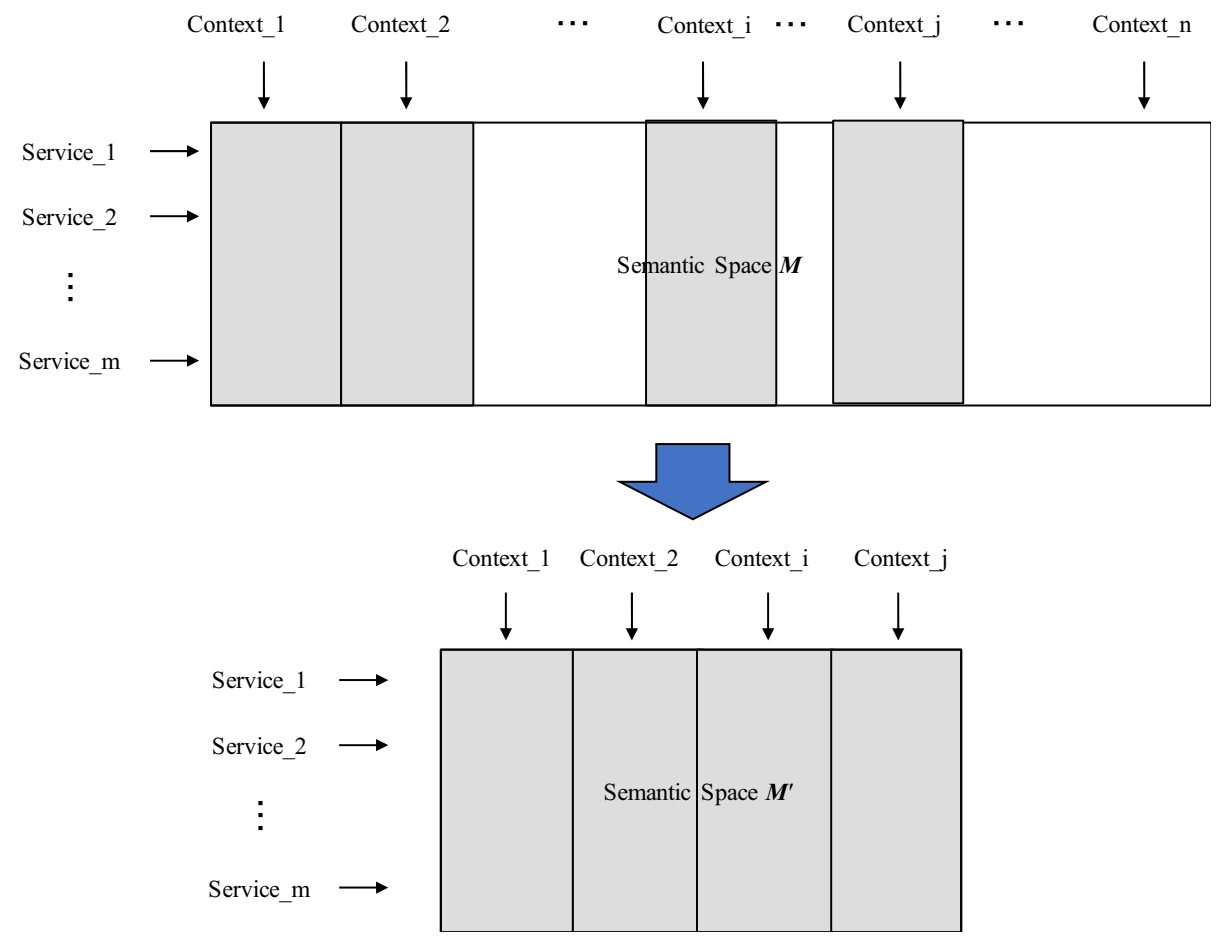

Figure 8. Semantic Space $\boldsymbol{M}^{\prime}$ 


\subsection{Correlation metric on Passenger's Semantic Subspace $\boldsymbol{M}^{\prime}$}

The similarities of the passenger's context vector ${\overrightarrow{v^{\prime}}}_{u}$. and service vectors ${\overrightarrow{v^{\prime}}}_{\text {service_i } i}$ are computed as follows (cosine similarity) in the semantic subspace $\boldsymbol{M}^{\prime} \cdot{\overrightarrow{v^{\prime}}}_{u}$ and ${\overrightarrow{v^{\prime}}}_{\text {Service_i } i}$ are given as follows:

$$
\begin{gathered}
{\overrightarrow{v^{\prime}}}_{\text {service } i}=\left\{x_{i 1}, \cdots, x_{i j}, \cdots, x_{i \cdot} \mid x_{i j} \in M^{\prime}\right\} \\
{\overrightarrow{v^{\prime}}}_{u^{\prime}}=\left\{y_{1}, \cdots, y_{k}, \cdots, y_{. \prime} \mid y_{k} \in \vec{v}_{u^{\prime}}, y_{k} \geq \text { threshold }|\varepsilon|\right\}
\end{gathered}
$$

The similarity between ${\overrightarrow{v^{\prime}}}_{u}$. and ${\overrightarrow{v^{\prime}}}_{\text {service_i } i}$ is computed as follows (cosine similarity):

$$
\operatorname{sim}\left({\overrightarrow{v^{\prime}}}_{u^{\prime}},{\overrightarrow{v^{\prime}}}_{\text {service }_{i}}\right)=\frac{\vec{v}_{u} \cdot \vec{v}_{\text {serivice } i} i}{\left|\overrightarrow{v_{u}} \cdot\right| \times\left|\vec{v}_{\text {service_ } \_}\right|}
$$

\subsection{Spatio correlation metric}

In this paper, we propose a method to measure the positional correlation between users and facilities/stores on the same floor. The measurement is obtained by filtering the area to be searched and measuring the physical distance (Figure 9). The filtering operation is realized by specifying the inside and outside of the ticket gate. The measurement of the physical distance sets the value range from 0 to 1 . For this purpose, the following formula is applied (7), where a represents the distance that exists in the same place as the user.

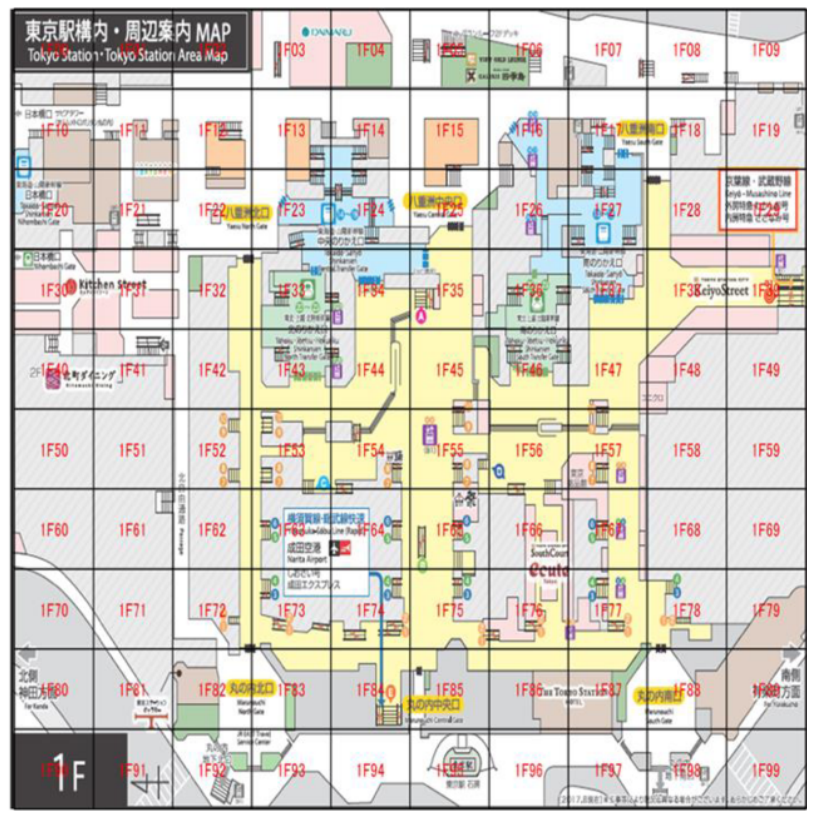

Figure 9. Spatio Mesh of Tokyo Station 


$$
\begin{aligned}
& \operatorname{sim}\left(u l_{\text {user }}, u l_{\text {service }_{i}}\right)=\frac{\log _{a}(a)}{\log _{a}\left(\left|u l_{\text {user }}-u l_{\text {service }_{i}}\right|\right)} \\
& \left|u l_{\text {user }}-u l_{\text {service }_{i}}\right|=\left\{\begin{aligned}
\left|u l_{\text {user }}-u l_{\text {service }_{i}}\right|, & \left|u l_{\text {user }}-u l_{\text {service }_{i}}\right| \geq a \\
a, & \left|u l_{\text {user }}-u l_{\text {service }_{i}}\right|<a
\end{aligned}\right.
\end{aligned}
$$

\subsection{Semantic Anticipation of Needs}

In this paper, the integrated correlation (IC) among Intention, Sense, and Distance is defined by an expression that multiplies each weight.

$$
\text { IC }=\operatorname{sim}\left(\vec{v}_{u c}, \vec{v}_{\text {service }_{i}}\right) \times \operatorname{sim}\left(\vec{v}_{\text {us }}, \vec{v}_{\text {service }_{i}}\right) \times \operatorname{sim}\left(u_{\text {user }}, u l_{\text {service }_{i}}\right)
$$

\subsection{Ranking}

We rank the services by the integrated correlation (IC). The rankings are displayed on a smartphone display and information display.

\section{System}

In this study, we constructed a system that provides facility/store information about the concourse on the first floor of Tokyo Station according to the context of the user. Figure 10, Figure 11, and Figure 12 show the system configuration diagram, class diagram, and ER diagram.

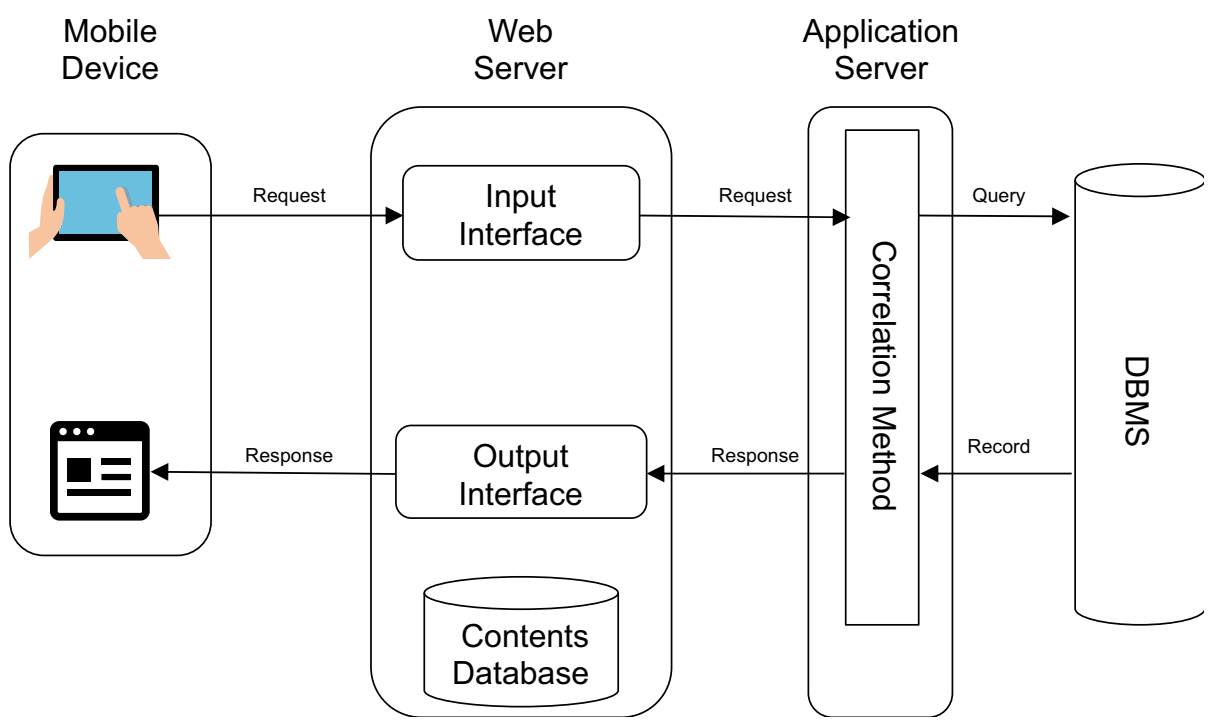

Figure 10. Architecture of Prototype System 


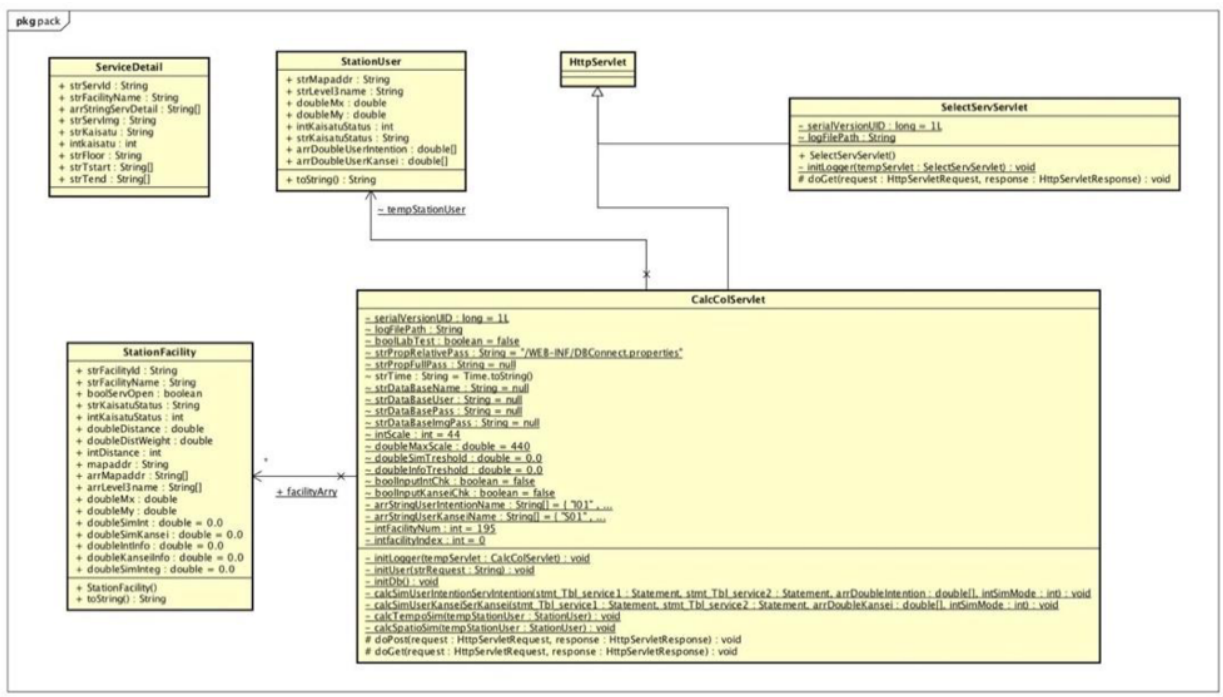

Figure 11. Class Diagram of the System

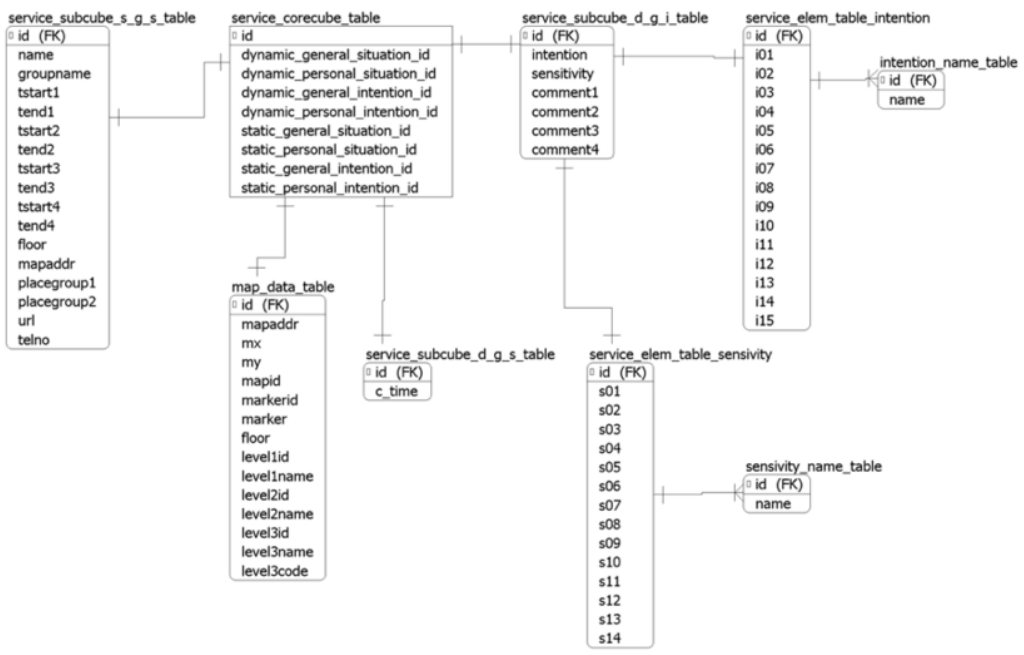

Figure 12. ER Diagram of the Database

The flow of use is listed as follows.

- The user selects his/her current position, search area (inside/outside the ticket gate), purpose, and sense in the input interface (Figure 13).

- The system computes the user input and the measurement of the correlation between stores and facilities. 
- The system displays a store/facility ranking list based on the correlation amount and a map that shows their locations on the output interface (Figure 14).

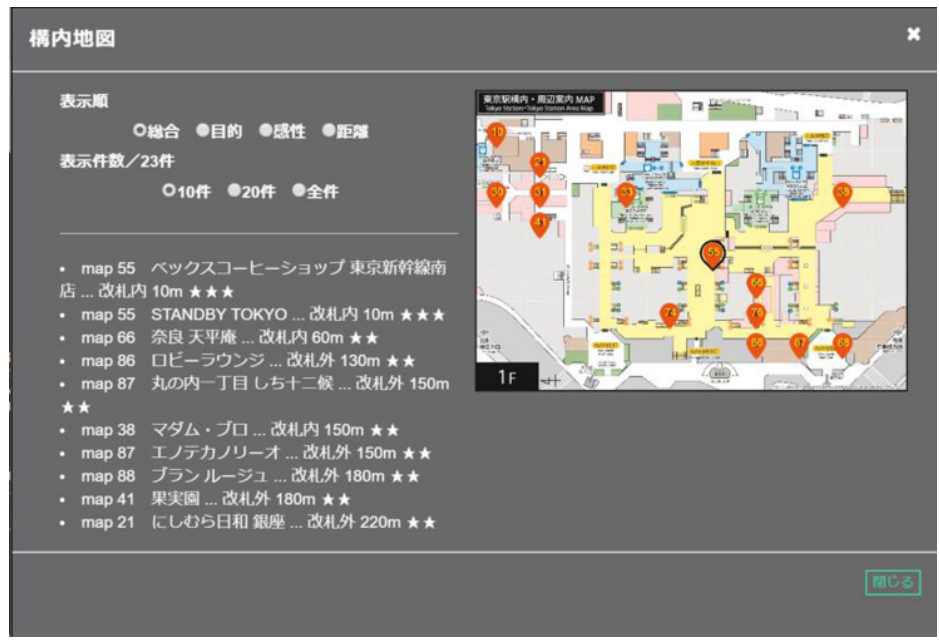

Figure13. Input Interface

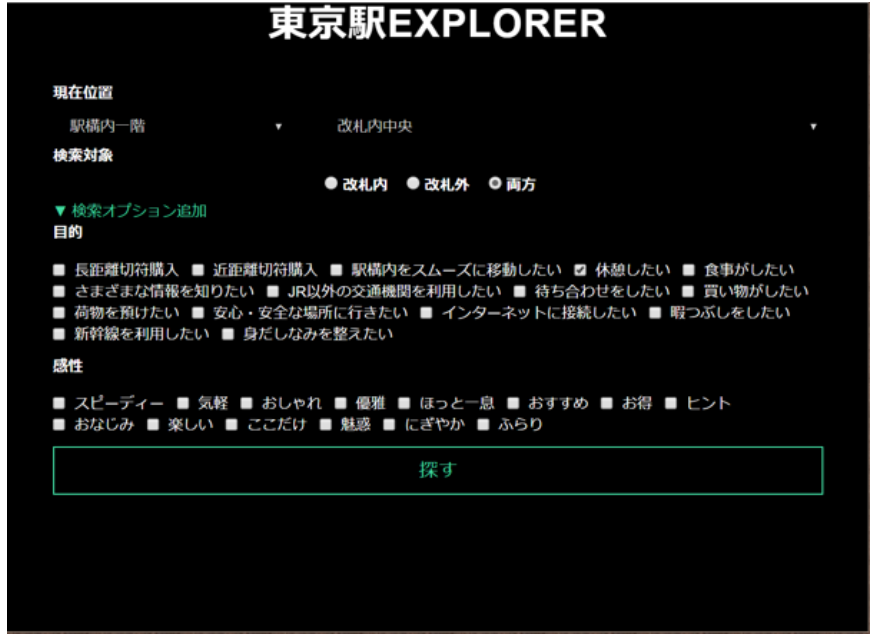

Figure14. Output Interface

\section{Experiment}

We conduct an experiment to verify that the method of measuring the correlation between the user context and facilities/stores proposed in this study is effective for station guidance. Specifically, a prototype system is employed in Tokyo Station to evaluate the usability and information acquisition load. The details of the implementation are shown as follows:

- Implementation period

May 16, 2018-May 25, 2018 
- Implementation location

Three locations in Tokyo Station (near the central passage north ticket gate, near the Marunouchi north gate, and near the Marunouchi central gate)

- Number of subjects

18

- Evaluation method

The subject was presented with a usage scene of Tokyo Station and a search task, and an information search was performed. The subject was asked to answer questionnaires, such as the System Usability Scale and User Experience evaluation (Figure 15, Figure 16) [15, 16].

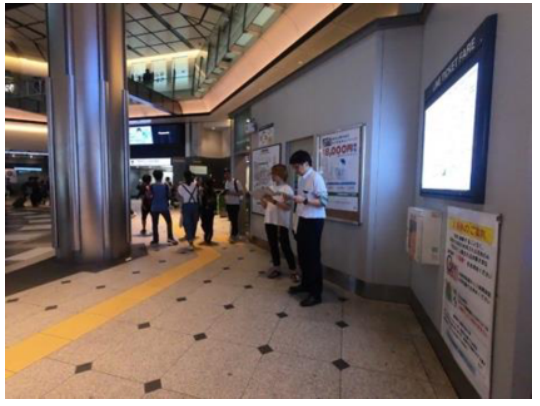

Figure 15. Experiment at Tokyo Station

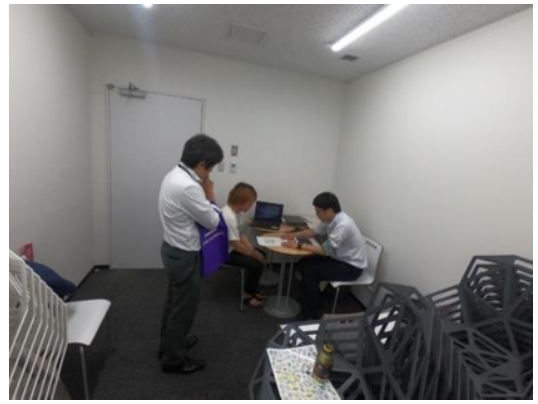

Figure 16. Questionnaire answer scene

\subsection{Correlation Calculation result}

The results of the correlation metric in the experiment are shown. The number of stores and facilities targeted by the constructed system is 300 , and those with a correlation of 0.1 or greater will be displayed in the ranking list.

\{"location":"1F38","intention": \{"I01":1\},"kansei": \{"S01":1,"S04":1\},"kaisatu ":"0","threshold":"0.1"\}

Figure 17 shows a parallel coordinate diagram of spatio correlation, intention correlation, and sensitive correlation. Figure 18 shows the integrated correlations of each store/facility. Figure 19 shows a visualized structure of the relationship between a user context and a store or a similar structure based on the integrated correlation amount. Gephi was employed as a visualization tool. In addition, a node arrangement algorithm is applied to understand the structure [17]. A user input example is provided as follows:

\{"location":"1F38","intention": \{C1\},"sense": \{C16\},"Gate": Inside and Outside"\} 


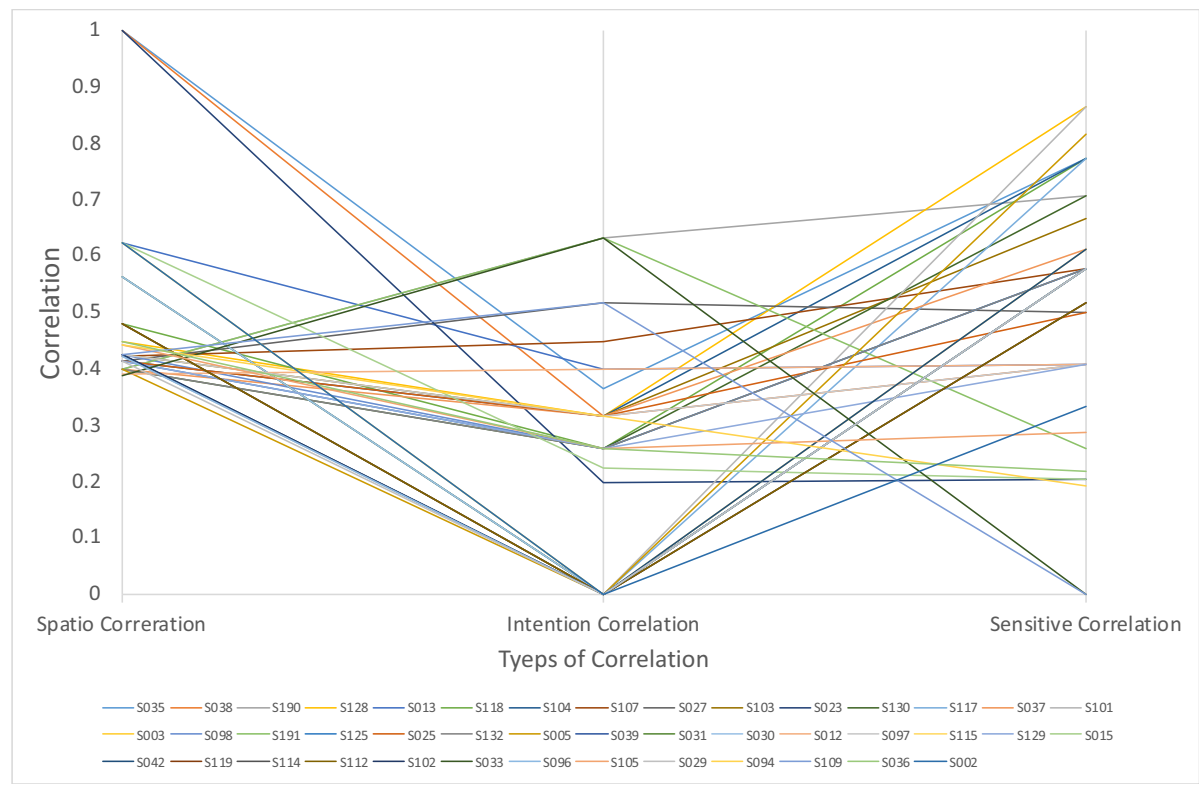

Figure 17. Parallel Coordinate Diagram

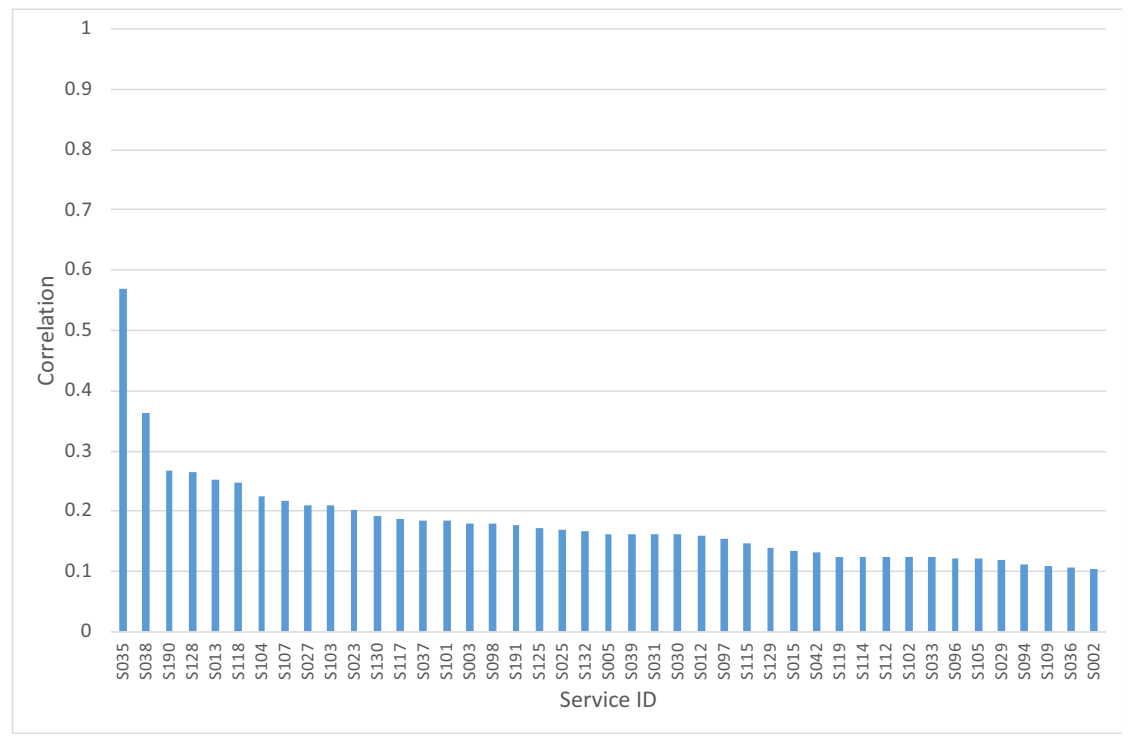

Figure 18. Total Correlation 


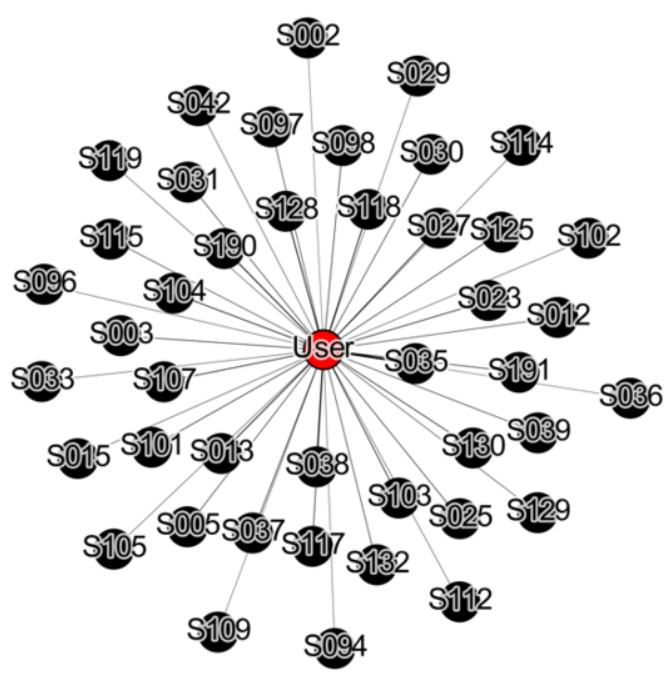

Figure 19. Context Cube Network of Correlation Results

\subsection{Usability evaluation questionnaire results}

Items of the questionnaire results and tabulation results are shown in Figure 20. The evaluation revealed that more than $70 \%$ of the participants wanted to use the prototype system, which confirms the effectiveness of the prototype system for information retrieval. More than $80 \%$ of users who utilize the system for the first time answered "I thought I need to learn a lot before using this system", "I do not think so or at all". This finding confirmed that the system was very effective.

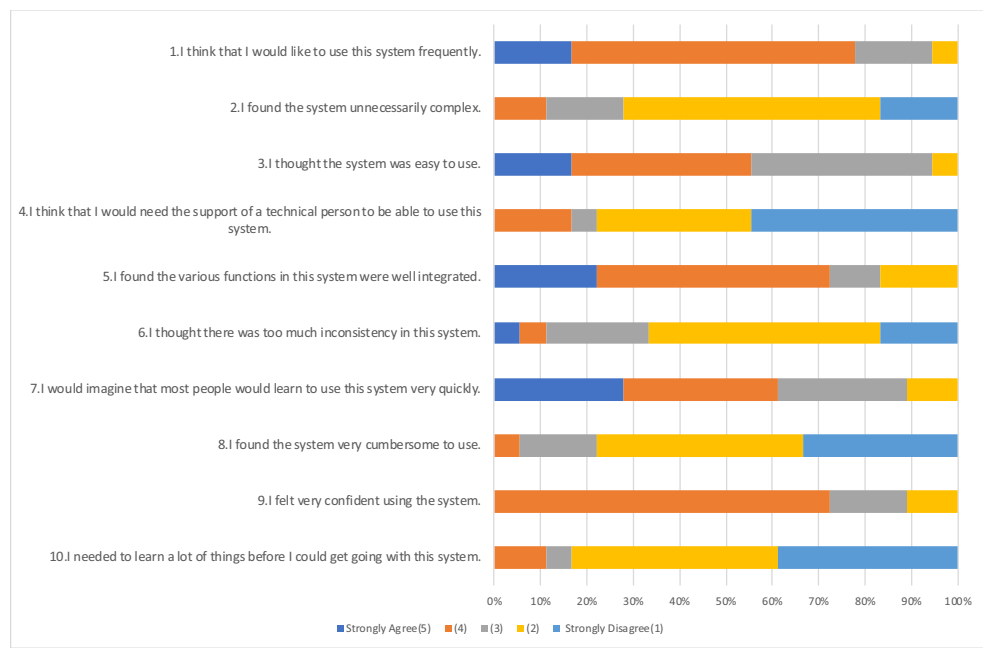

Figure20. Evaluation results of usability (18 subjects) 


\subsection{Evaluation of purpose and emotional search method}

Figure 21 shows the evaluation of items related to purpose and sense search methods. For all items, "Strongly agree" and "Agree" exceeded $60 \%$, which indicates the effectiveness of the proposed method. In particular, the items of "This search method has potential" and "I want to use this search method in town" accounts for more than $80 \%$. The proposed method is novel, and discovering the service consumption of users is possible. In addition, more than $70 \%$ of participants respond "I would like to recommend this search method to my friends." and "I want to use this search method frequently", which confirms that the user's search needs can be satisfied.

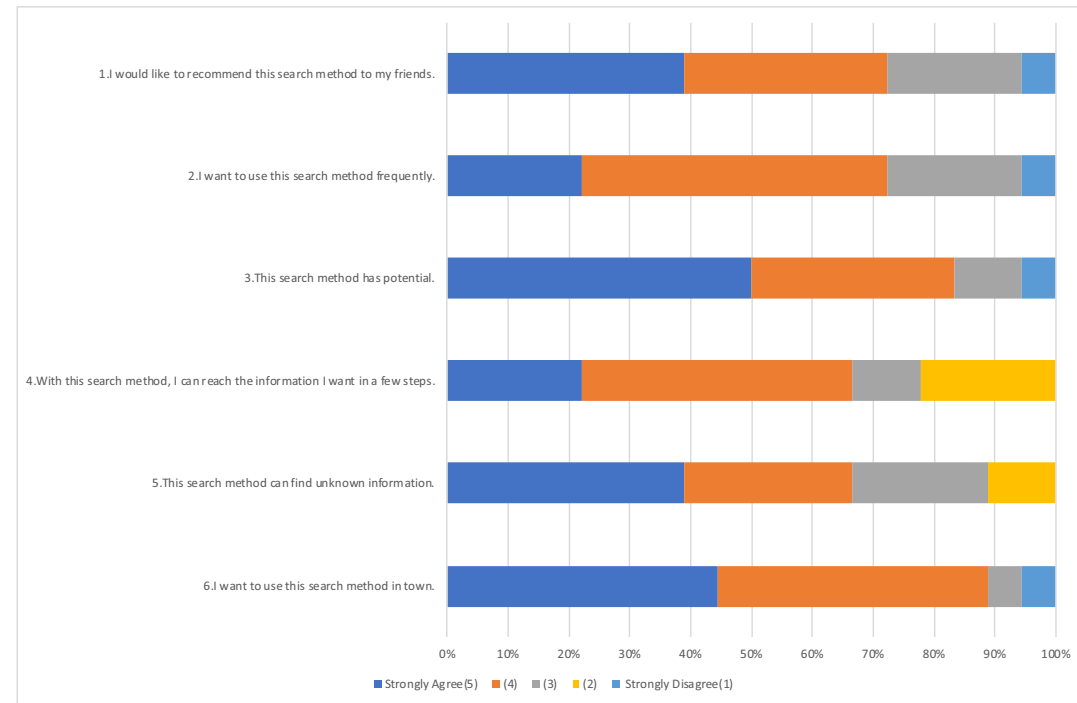

Figure21. Evaluation of purpose and sense search method (18 subjects)

\section{Conclusions and Future Work}

In this study, we proposed a data model, the "Context Cube Semantic Network Model", that determines the user's context and facilities and stores in the station, and a measure that integrates the user's intention, sensitivity, and location with the correlation amount among the Context Cubes. We implemented a computing method for the station guidance system and allowed the subjects to use the system in the station to verify the effectiveness of the method.

For the usability evaluation, we obtained the evaluation value for usability and user experience. As a result, more than $60 \%$ of the participants answered positively about the ease of information acquisition, and more than half of the participants answered positively about the usability. Therefore, the proposed method was effective for information acquisition in the station yard. Since more than half of the subjects would like to introduce this search method to friends and others, communication to introduce the method will likely be induced. In addition, half of the participants indicated new 
discoveries. For this reason, we believe that more meaningful support for using the station premises is possible.

However, some subjects did not respond positively because they did not know which purpose/sensitivity item to choose and the ranking results did not show their desired store. Therefore, consider the function that supports the selection of items and the output of the ranking results that are more suited to the context of the user.

In the future, we will acquire a substantial amount of data about the situation, purpose, and sensibility when using the store and investigate the application of judgment algorithms, such as machine learning, to improve the user's station and railway experience.

\section{References}

[1] Yasushi Kiyoki, Takashi Kitagawa, Takanari Hayama, “A metadatabase system for semantic image search by a mathematical model of meaning," ACM SIGMOD Record, vol.23, no.4, pp.34-41, 1994.

[2] Yasushi Kiyoki, Shiori Sasaki, Nhung Nguyen Trang, Nguyen Thi Ngoc Diep, “Cross-Cultural Multimedia Computing with Impression-Based Semantic Spaces," Conceptual Modelling and Its Theoretical Foundations, Lecture Notes in Computer Science, Springer, March 2012, pp.316-328.

[3] Yasushi Kiyoki, Xing Chen, "Contextual and Differential Computing for the Multi-Dimensional World Map with Context-Specific Spatial-Temporal and Semantic Axes," Information Modelling and Knowledge Bases, vol.XXV, IOS Press, pp.82-97, 2014.

[4] Motoki Yokoyama, Yasushi Kiyoki, Tetsuya Mita, "Similarity-Ranking Method based on Semantic Computing for a Context-Aware System", The Fifth International Conference on Knowledge Creation and Intelligent Computing, Manado, Indonesia, November 15-17, 2016.

[5] Motoki Yokoyama, Yasushi Kiyoki, Tetsuya Mita, "A similarity-ranking method on semantic computing for providing information-services in Station-Concierge System", EMITTER International Journal of Engineering Technology, Vol.5 No1, June 2017.

[6] Motoki Yokoyama, Yasushi Kiyoki, Tetsuya Mita, "A Correlation Computing Method for Integrating Passengers and Services in Semantic Anticipation", INFORMATION MODELLING AND KNOWLEDGE BASES XXX, 2019.

[7] Mikiko Sakamoto, "Technical Innovation in Railway Service: The JR East App," JR East Technical Review, Report number: No. 28-Spring, 2014.

[8] Takeshi Nakagawa, “ICT-Based Information Services for Customers," JR East Technical Review, Report number: No. 28-Spring, 2014.

[9] Kaoru Mori, Shuichi Kurabayashi, Naoiki Ishibashi, Yasushi Kiyoki, “An Active Information Delivery Method with Dynamic Computation of Users' Information in Mobile Computing Environments," Proceedings of Data Engineering Workshop2004,1-A-04, 2004.

[10] Gregory D. Abowd, Anind K. Dey, Peter J. Brown, Nigel Davies, Mark Smith, Pete Steggles, "Towards a Better Understanding of Context and Context-Awareness," First International Symposium on Handheld and Ubiquitous Computing(HUC'99), Germany, pp. 304-307, 1999.

[11] Motoki Yano, Katsuhiko Kaji, Nobuo Kawaguchi, “App.Locky: Users' Context Collecting Platform for the Context-aware Service Recommendation," Journal of Information Processing ,52(12) 3274-3288, 2011.

[12] Lina Yao, Quan Z. Sheng, Yongrui Qin, Xianzhi Wang, Ali Shemshadi, and Qi He," Context-aware Point-of-Interest Recommendation Using Tensor Factorization with Social Regularization", SIGIR '15: Proceedings of the 38th International ACM SIGIR Conference on Research and Development in Information RetrievalAugust 2015 Pages 1007-1010.

[13] Stathis Maroulis, Ioannis Boutsis, Vana Kalogeraki, “Context-Aware Point of Interest Recommendation using Tensor Factorization”,2016 IEEE International Conference on Big Data (Big Data).

[14] Daniel Kahneman, "Thinking, Fast and Slow", Farrar, Straus \& Giroux Inc, 01 Sep 2017.

[15] Brooke, John, "SUS'A quick and dirty' usability scale", Usability Evaluation in Industry, Taylor \& Francis, 1996.

[16] Masaya Ando, "User Experience Design TextBook", Maruzen Publishing Co.,LTD, 2016(Japanese).

[17] Tokyo station city, Tokyo station city, http://www.tokyostationcity.com/, Retrieved January $26^{\text {th }} 2018$.

[18] https://gephi.org/ 